\title{
What Barriers Exist in the Minds of Vaccine-Hesitant Parents, and How Can We Address Them?
}

Andrew R. Kerrigan, MD, MPH; Imane Aitnouri; Jessica Mar; Wayne Altman, MD

\begin{abstract}
BACKGROUND AND OBJECTIVES: Despite substantial health benefits and prolific research efforts to demonstrate safety and increase uptake, vaccine hesitancy has increased dramatically. This study aimed to systematically analyze available literature on vaccine hesitancy in the United States and determine the rationale behind vaccine-hesitant parents and potential interventions.
\end{abstract}

METHODS: We conducted a literature search and identified 232 articles; we included 90 after screening. We pulled information from each article using standardized questions for "type of study," "population," "specific vaccine," "reasons for hesitancy," "hesitancy prevalence," "attempt at change," "results of intervention," and "future interventions." We created recurrent themes from the data and analyzed these themes via descriptive statistics.

RESULTS: Vaccine safety was the most commonly identified reason for vaccine hesitancy amongst studies (50\%), followed by not enough information $(30 \%)$, side effects $(26 \%)$, low risk of disease $(26 \%)$, social norms $(22 \%)$, vaccine schedule (21\%), not recommended by doctor (21\%), efficacy $(18 \%)$, cost/ access (13\%), sexual concerns (12\%) and distrust of establishment (7\%). Only $20 \%$ of papers documented an intervention, and $<50 \%$ of these reported increased vaccination rates or intent. More research was the most commonly identified next step (49\%).

CONCLUSIONS: Despite large amounts of research on vaccination rates, patients are still reporting not enough information and safety as the most common reasons for vaccine hesitancy. Interventions were few and without promising results. More research was the most suggested intervention. Such research must address concerns of the vaccine-hesitant community, comparing risks and benefits of each vaccination in a longitudinal, coherent, and transparently unbiased fashion.

(Fam Med. 2020;52(9):626-30.)

doi: 10.22454/FamMed.2020.432940

$\mathbf{V}$ accines have inarguably produced substantial public health benefits. Recently, however, parents' confidence in vaccination against preventable diseases has been wavering. The prevalence of concerns over vaccination (vaccine hesitancy) increased from 19\% in 2000 to $50 \%$ in $2009 .{ }^{4}$ Vaccine children, and undoubtedly played measles in 20 years. ${ }^{4}$ The problem also exists in Europe, where they experienced a fourfold increase in measles from 2017 to 2018, with more than 20,000 cases. $^{5}$
Instead of vilifying and shaming parents who hesitate to vaccinate their children, we should try to understand what the common barriers are, and how we can effectively address them. Much research has been undertaken to determine the reasons behind vaccine hesitancy, but these studies have produced a wide range of results that can be difficult to process. With this review, we aimed to assess the available literature and condense the information into major unifying themes. A properly synthesized data set could provide a clear avenue for understanding the major rationales of vaccine-hesitant parents and suggest interventions that may increase vaccine confidence in parents.

\section{Methods}

Our team identified original research that addressed parental vaccine beliefs. Specifically we sought articles that described the reasons for vaccine hesitancy, and any interventions that targeted these reasons. We searched PubMed using the MeSH terms "United States," "parents," "vaccines," and "attitudes to health," and identified 232 articles. After screening, we included a total of 90 articles in the final analysis (Figure 1). Three authors used a part in the highest US incidence of
From the Department of Family Medicine (Dr Altman and Ms Aitnouri), Tufts University (Ms Mar) School of Medicine (Dr Kerrigan), Boston, MA. 
uniform template of data points and questions to keep the data extraction systematic and to mitigate bias (Table 1). If there was any uncertainty about a specific data point, the decision was made by consensus.

Our analysis of the reasons for vaccine hesitancy consisted of the identification of recurrent themes in the data. Themes were derived from the papers themselves, not from predetermined categories. Each author independently developed a list of themes, then the team determined the final categorization, with as little overlap as possible and without excluding any identified themes. To ensure accuracy and internal validity, each of the final categories was independently analyzed twice by two separate individuals. Disputes between the two analyses were settled by a third reviewer. If other themes became apparent, the themes were recreated, and the data set was reanalyzed. We analyzed data using descriptive statistics.
Results

Reported Reasons for Vaccine

Hesitancy

Eighty-two of the 90 included papers reported on reasons for vaccine hesitancy (Table 2). The other eight studies did not identify reasons for hesitancy; most were intervention studies. Vaccine safety was cited by half of the papers (41) as a concern. Not having enough information was the second most common reason, reported in 25 articles.

Figure 1: The PRISMA Flow Diagram for the Literature Search Conducted for This Study

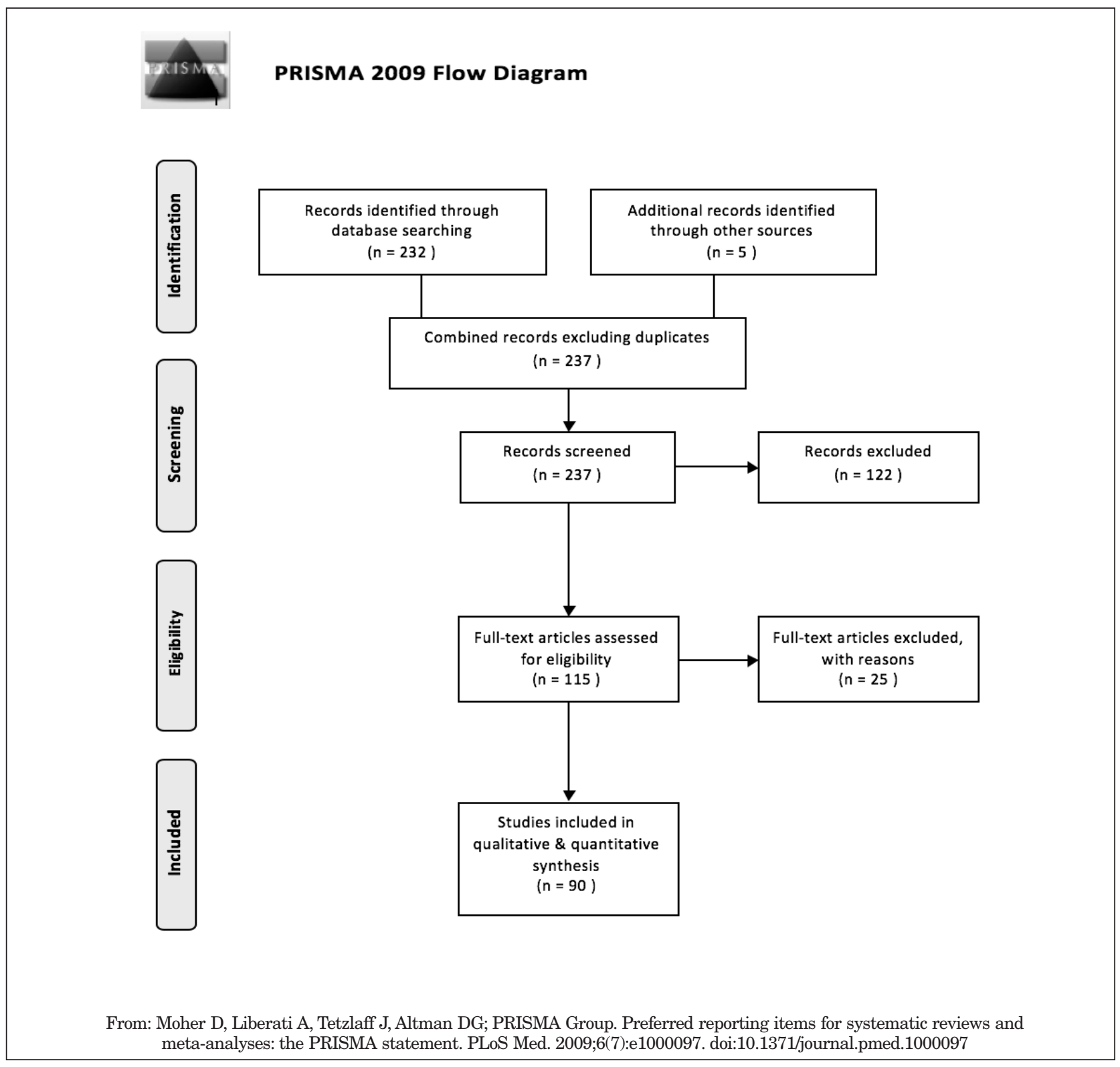


Table 1: Templated Questions Used for Standardized Data Extraction From Each Article Included in the Study

\begin{tabular}{|l|}
\hline Types of studies: What type of study was it? Did the study include quantitative and/or qualitative data? \\
\hline Population: Who was study population, including location, socioeconomic factors, and other demographics? \\
\hline Specific vaccine: Did the study focus on vaccine hesitancy related to specific vaccines or vaccines in general? \\
\hline Reasons for hesitancy: What reasons did the study population state contributed to vaccine hesitancy? \\
\hline $\begin{array}{l}\text { Hesitancy prevalence: Did the article note what percentage of parents identified the reason as a cause of vaccine hesitancy, } \\
\text { or did the article identify a majority reason? }\end{array}$ \\
\hline Attempt at change: Did the article include an intervention aimed at targeting a reason for vaccine hesitancy? \\
\hline Results of intervention: Did the article report the results of an intervention and, if so, what were they? \\
\hline Suggested future interventions: Did the article identify any suggestions for what future interventions might be effective? \\
\hline
\end{tabular}

Table 2: Recurrent Themes for Hesitancy Identified in the Literature and the Frequency Which They Were Identified Across Papers

\begin{tabular}{|l|c|}
\hline \multicolumn{1}{|c|}{ Reasons for Hesitancy } & Number of Articles* \\
\hline Safety/risk & $41(50 \%)$ \\
\hline Not enough information & $25(30 \%)$ \\
\hline Side effects & $21(26 \%)$ \\
\hline Low risk of disease & $21(26 \%)$ \\
\hline Social norms ${ }^{\dagger}$ & $18(22 \%)$ \\
\hline Vaccine schedule & $17(21 \%)$ \\
\hline Not recommended by doctor & $17(21 \%)$ \\
\hline Efficacy & $15(18 \%)$ \\
\hline Cost and access & $11(13 \%)$ \\
\hline Sexual concerns & $10(12 \%)$ \\
\hline Distrust of establishment & $6(7 \%)$ \\
\hline
\end{tabular}

$* \mathrm{~N}=82$.

${ }^{\dagger}$ Feeling influenced by a community of like-minded vaccine-hesitant persons, religion/culture, or parents/family

* Believing that that the vaccine would increase promiscuity and/or thoughts that the vaccine could alter sexual or reproductive functioning

\section{Attempts at Change}

Only 18 papers (20\%) documented an actual attempt at change. Of these, $10(56 \%)$ were educational interventions on risks/benefits, four focused on changing the schedule of vaccinations, three implemented school-based programs, two addressed social norms with culturally tailored messages, and two were interventions at the physician level. Group counseling sessions (to discuss vaccine hesitancy with doctors and peers) and combination vaccines were addressed by one study each.
Suggested Future Interventions

The most cited recommendation to reduce vaccine hesitancy was to obtain more research (49\%), though $34 \%$ of papers concluded that better physician recommendations would decrease vaccine hesitancy, and $28 \%$ stated that leveraging social norms would be beneficial. Stressing the benefits of vaccination was more often cited (13\%) than stressing the risks of nonvaccination (8\%, Table 3$)$.

\section{Discussion}

As anticipated, our analysis yielded a wide variety of studies with various designs, results, and conclusions. Despite this, several themes recurred. From these recurrent themes, commonalities across the individual data sets emerged.

Concern about safety was the most frequently cited reason for vaccine hesitancy. Papers often reported on safety in general, identifying some specific safety concerns, but rarely reporting supporting data or prevalence. Focus group data from a 2017 study revealed a breadth of safety concerns that vaccine-hesitant parents shared. These concerns included several themes: (1) possible long-term effects of vaccines (asthma, allergy, obesity, childhood cancer, SIDS, autism), (2) the number and timing of vaccines (too many vaccines in the first 15 months and too many vaccines given simultaneously), (3) other ingredients in the vaccines (toxins, heavy metals such as mercury and aluminum), and (4) 
Table 3: Different Suggestions for Future Interventions Reported in the Literature and the Frequency Which They Were Suggested Across Papers

\begin{tabular}{|l|c|}
\hline \multicolumn{1}{|c|}{ Intervention } & Number of Articles* \\
\hline More research & $42(49 \%)$ \\
\hline Better physician recommendations & $29(34 \%)$ \\
\hline Establish norms & $24(28 \%)$ \\
\hline Stress benefit & $11(13 \%)$ \\
\hline Stress risk & $7(8 \%)$ \\
\hline Improve access & $7(8 \%)$ \\
\hline Flexible schedules & $6(7 \%)$ \\
\hline Combo vaccines & $6(7 \%)$ \\
\hline Legal/mandates & $5(6 \%)$ \\
\hline Present unbiased risk & $3(4 \%)$ \\
\hline
\end{tabular}

$* \mathrm{~N}=85$.

the underreporting of side effects by vaccine manufacturers. ${ }^{6}$

Despite a large body of literature, not enough information was the second most commonly cited reason. This could be because many studies that cited safety as a concern focused on one specific vaccine instead of vaccines in general. Mistrust in the existing literature could also contribute to safety concerns, as distrust of establishment was another reason for hesitancy.

Not recommended by doctor was another surprisingly common theme. The reasons behind this are unclear, given the strong support of vaccination by the medical community. One possibility is that doctors are not adequately engaging patients after an initial answer of "no," and are neglecting patients who might consider vaccination with continued counseling. This is supported by our findings that studies grossly excluded delayed and partial vaccination as an option. All evidence clearly supports the Advisory Committee on Immunization Practices vaccination schedule, and physicians should strongly recommend this as the only evidencebased vaccine schedule. ${ }^{7}$ However, we should continue dialogue with parents refusing vaccination, as delayed or partial schedules are better than no vaccination and establishes a rapport that may allow for increased vaccination in the future.
Doctors may also feel they don't have enough information. While the American Academy of Pediatrics and Centers for Disease Control and Prevention do give guidance on general approaches to these conversations, keeping up with hard data and multiple studies on various vaccinations can be tedious and is not realistic for

The theme of not enough information is highlighted by more research being cited as the most commonly proposed future intervention. This matches up with vaccine-hesitant parents' desire to know more about vaccines. The second most common next step, proposed by more than one-third of papers, was to improve physician recommendations. Further research must be done to determine why physicians are not recommending vaccinations or are not presenting the information effectively.

The paucity of intervention studies suggests that there is no clear consensus on an approach to addressing vaccine hesitancy. Despite this, most intervention studies attempted to educate patients. Only two interventions attempted to address physicians' recommendations, which is disproportionate to the number of studies citing this problem. One likely explanation for this is that researchers don't have a solid understanding of why physician recommendations are lacking. As many doctors. ${ }^{8,9}$ previously discussed, one possibility may be that physicians lack the appropriate information to engage in a meaningful conversation about vaccine hesitancy and therefore veer away from intelligent debate. Another possibility is that doctors would rather not believe that they are contributing to the vaccine hesitancy movement.

The results of these interventional studies show that their impact is minimal overall. Although more than two-thirds reported having had some impact, less than 50\% reported an improvement in vaccination behavior, perception, or intent. The most common intervention - educationproduced an increased intention to vaccinate in five of nine studies, but only one reported an increase in vaccination rates. Two showed poor vaccination rates, even after the education intervention. ${ }^{6,10}$ One study found that an increase in intent did not translate to an increase in vaccination rates. ${ }^{11}$ This suggests that patient education and other interventions, as of now, are largely ineffective.

\section{Recommendations}

Since the current interventions do not effectively address parents' concerns about vaccine safety or their perceived lack of information, in the future we must focus on building an evidence base that is accessible and credible to them. To this end, we suggest a large long-term cohort study of patients who are unvaccinated, partially vaccinated, delayed vaccinated, and fully vaccinated. The advisory board should include members of the vaccine-hesitant community, as our research suggests that social norms are important to the vaccine hesitancy movement. The study would also benefit from the absence of pharmaceutical funding, as distrust of the establishment has been noted in several papers. Finally, and perhaps most importantly, the proposed study should attempt to answer the questions that the vaccine-hesitant community is asking. The current research satisfies the 
medical community but does not effectively address the concerns of vaccine-hesitant parents. A single collaborative research effort that leverages the power of electronic health records could finally meet the needs of both the medical and vaccine-hesitant communities.

\section{Conclusions}

By unifying these data, we now have a better representation of the reasons for vaccine hesitancy in the United States. Our analysis supports the widespread beliefs that vaccine hesitancy is driven by a multitude of factors, with the most concerns over the safety of the vaccines and not being satisfactorily informed about the health risks and benefits related to vaccination. It's likely that feelings of not having enough information reflect a distrust of the currently available data and drive the concerns over health outcomes. Although our research indicates that current physician recommendations to vaccinate are inadequate, we also learned that patient self-education with the available data has mixed results and is not consistently effective. While we wait for more and better studies on how to overcome parents' vaccine hesitancy, the best approaches seem to be to recommend the appropriate vaccines at every visit, regardless of previous uptake; offer vaccination scheduling options; and stress the benefits of vaccination rather than the risks of nonvaccination.

CORRESPONDING AUTHOR: Address correspondence to Dr Andrew R. Kerrigan, Tufts University School of Medicine, 145 Harrison Avenue, Boston, MA 02111. 617-636-0432. AndrewKerriganTufts@gmail.com.

\section{References}

1. Greenwood B. The contribution of vaccination to global health: past, present and future. Philos Trans R Soc Lond B Biol Sci.:20130433.

2. Roser M, Ritchie H, Dadonaite B. Child and infant mortality. Our World in Data. https://ourworldindata.org/child-mortality. Published 2013. Accessed July 15, 2020.

3. Heron M. Deaths: leading causes for 2016. Natl Vital Stat Rep. 2018;67(6):177. https://www.cdc.gov/nchs/data/nvsr/ nvsr67/nvsr67_06.pdf. Accessed July 15, 2020.

4. Smith TC. Vaccine rejection and hesitancy: A review and call to action. Open Forum Infect Dis. 2017;4(3):ofx146.

5. Europe observes a 4-fold increase in measles cases in 2017 compared to previous year [Press release]. Copenhagen, Denmark: World Health Organization, Regional Office for Europe; February 19, 2018. http://www.euro.who.int/en/ media-centre/sections/press-releases/2018/ europe-observes-a-4-fold-increase-inmeasles-cases-in-2017-compared-toprevious-year. Accessed January 21, 2019.

6. Potter N, Quinn M, Herring M, Altman W. Taking a shot at immunization adherence. PRiMER Peer-Rev Rep Med Educ Res. 2017;1:15.
7. Centers for Disease Control and Prevention. Recommended child and adolescent immunization schedule for ages 18 years or younger, United States, 2020. https:// www.cdc.gov/vaccines/schedules/hcp/ $\mathrm{imz} /$ child-adolescent.html. Accessed July 15, 2020.

8. American Academy of Pediatrics. Vaccine Hesitant Parents. https://www.aap.org/ en-us/advocacy-and-policy/aap-healthinitiatives/immunizations/Pages/vaccinehesitant-parents.aspx. Accessed July 15, 2020.

9. Centers for Disease Control and Prevention. Provider Resources for Vaccine Conversations with Parents. Retrieved from https://www.cdc.gov/vaccines/hcp/ conversations/index.html. Published November 30, 2015. Accessed July 15, 2020.

10. Kepka D, Coronado GD, Rodriguez HP, Thompson B. Evaluation of a radionovela to promote HPV vaccine awareness and knowledge among Hispanic parents. J Community Health. 2011 9;36(6):957-965.

11. Iroh Tam PY, Visintainer P, Fisher D. Response to an education program for parents about adult pertussis vaccination. Infect Control Hosp Epidemiol. 2009;30(6):589592. 